\title{
A STUDY ON YIELD AND USEFULNESS OF NON-CONTRAST CT BRAIN IMAGING IN ACUTE STROKE AT A TERTIARY CARE INSTITUTE IN SOUTH INDIA
} \begin{abstract}
Gautham Chinnathambi Somanathan6, Prakash Subramanian7, Sindhiya Jayachandran ${ }^{8}$
1 Professor and HOD, Department of Internal Medicine, Govt. Stanley Medical College and Hospital.

2 Professor, Department of Internal Medicine, Govt. Stanley Medical College and Hospital.

3 Professor and HOD, Department of Radiology, Govt. Stanley Medical College and Hospital.

${ }_{4}^{4}$ Post Graduate, Department of Internal Medicine, Govt. Stanley Medical College and Hospital.

${ }^{5}$ Assistant Professor, Department of Internal Medicine, Govt. Stanley Medical College and Hospital.

${ }^{6}$ Assistant Professor, Department of Internal Medicine, Govt. Stanley Medical College and Hospital.

${ }^{7}$ Assistant Professor, Department of Internal Medicine, Govt. Stanley Medical College and Hospital.

${ }^{8}$ Post Graduate, Department of Internal Medicine, Govt. Stanley Medical College and Hospital.
\end{abstract}

Jayanthi Rangarajan'1, Vasumathi Govindaraj², Amarnath Chelladurai', Santhosh Kumar Vaseekaran', Ramalingam Arunachalam ${ }^{5}$,

ABSTRACT
BACKGROUND
Non-enhanced CT scanning of the head remains the first-line diagnostic test for the emergency evaluation of acute stroke
because of its speed, its convenient availability at most hospitals and its ability to sensitively depict intracranial haemorrhage.[1]
This is an observational study done to ascertain the yield and usefulness of non-contrast CT brain imaging in acute stroke in a
tertiary care centre.

\section{METHODS}

This was a prospective observational study done from June 2015 - November 2015 in a tertiary care centre. The study included 75 patients above 18 years of age who presented with any new-onset neurological deficit to our hospital. CT imaging was done for all those patients. Pregnant patients and those with previous neurological deficits were excluded from this study. A detailed study on the sex, age of the patient, time of presentation to our hospital, types of stroke along with site of involvement were studied. Data was recorded and analysed.

\section{RESULTS}

Amongst the 75 patients we studied 56 were females, 44 were males, $64 \%$ of our patients had infarct, $21 \%$ had haemorrhage, $19 \%$ of our patients had normal study at the time of presentation. Amongst those who had evidence of CT proven infarct, 3 patients presented to us within 6 hours, 6 patients between 6-12 hours, 26 patients between 12-24 hours, 10 patients after 24 hours. Amongst the $19 \%$ who had no evidence of stroke in imaging studies, $85 \%$ presented within 6 hours to our hospital.

\section{CONCLUSIONS}

Our study concluded that females are predominant in patients presenting with stroke, most common cause of stroke was infarct with capsuloganglionic region being the most common site of involvement and radiological yield of evidence of plain CT had positive correlation with advancing age of infarct.

\section{KEYWORDS}

Non-Contrast CT Brain, Acute Infarct, Haemorrhage.

HOW TO CITE THIS ARTICLE: Rangarajan J, Govindaraj V, Chelladurai A, et al. A study on yield and usefulness of non-contrast CT brain imaging in acute stroke at a tertiary care institute in South India. J. Evolution Med. Dent. Sci. 2016;5(38):2325-2328, DOI: $10.14260 /$ jemds/2016/540

\section{INTRODUCTION}

Stroke is a heterogeneous syndrome caused by multiple mechanisms, but all result in some disruption of CBF and subsequent tissue damage. Despite well-developed collateral circulation, there are regions of the brain that are more vulnerable to abnormal perfusion pressures. Generally, if the ischemia is sufficient to produce permanent damage, the brain tissue responds in a well-known fashion. The most sensitive brain cells to ischemia are the neurons followed by the astrocytes, oligodendroglia and microglia.

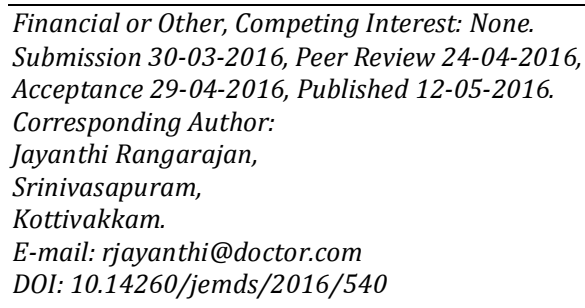

Earliest changes in neurons occur as quickly as $20 \mathrm{~min}$ after complete ischemia resulting in swollen, disorganized mitochondria. A failure of the ATP-driven sodium and potassium pump leads to increased intracellular water. Accumulation of glutamate has an effect on calcium channels also resulting in increasing levels of intracellular water. At 4$6 \mathrm{~h}$ post-ischemia the neurons begin to shrink, their nuclei stain more intensely and their cytoplasm becomes eosinophilic. The synaptic gaps enlarge with expansion of astrocytic end feet, synaptic encrustations and further shrinkage of neurons.

This process is usually completed by $24 \mathrm{~h}$ after ischemia. Macroscopically, visible infarcts are seen as areas of brain "softening" with loss of the borders between white and gray matter and focal swelling with effacement of the gyri. This swelling due to intracellular cytotoxic oedema is at a maximum between 24 and $48 \mathrm{~h}$. The reparative and resorptive mechanisms start at 24-48 $\mathrm{h}$, beginning at the periphery of the infarct and proceeding towards its centre. 
The late stages of infarcts are recognized by gliosis, encephalomalacic cysts, shrunken gyri (Ulegyria), enlarged sulci and adjacent dilatation of CSF-containing spaces. This final pathologic stage of infarctions is reached by 2-4 weeks, but resorption of the necrotic tissue may last for months.

Computed Tomographic (CT) images of the brain are produced by scanning a collimated beam of x-rays through the brain in thin, sequential slices. The $x$-ray output is counted, analysed and reconstructed for clinical interpretation. The newer generation scans use spiral technology, where the imaging is performed in a continuous helical fashion instead of the conventional slice-by-slice method. Non-contrast cranial Computed Tomography (CT) is the initial imaging method of choice for patients with acute onset of neurologic deficits, because it is readily available in most emergency departments, can be performed rapidly and is the best method to identify acute haemorrhage or other mimics of acute stroke.

The diagnosis of ischemic cerebral infarction by CT usually is not possible for $12-24 \mathrm{hr}$. after the event.[2] In essence in the first hours after onset of a neurologic deficit, we perform computed tomography to look for ischemic stroke. Its main limitation however is the limited sensitivity in the acute setting. Detection depends on the territory, the experience of the interpreting radiologist and of course the time of the scan from onset of symptoms. Non-Contrast CT (NCCT) remains the gold standard means of detecting intracranial haemorrhage in acute stroke. Blood is hyperdense because of its high electron density. As blood is broken down, density on CT declines by approximately 1.5 Hounsfield Units (HU) per day. Old haemorrhage appears hypodense on CT within a time scale determined by the volume of the initial haematoma. Small bleeds may be indistinguishable from infarcts within days of the event.

Imaging in patients with acute stroke should be targeted toward assessment of the four Ps-parenchyma, pipes, perfusion and penumbra-as described by Rowley. ${ }^{[3]}$ This approach enables the detection of intracranial haemorrhage, differentiation of infarcted tissue from salvageable tissue, identification of intravascular thrombi, selection of the appropriate therapy and prediction of the clinical outcome.

Because of its $24 / 7$ availability, CT maintains a primary role in the evaluation of patients with acute stroke and remains the "gold standard" for detection of cerebral haemorrhage. Timely diagnosis of cerebral infarction remains challenging and is even more crucial with the emergence of new therapies. The best time to image a stroke patient with CT is as soon as possible, although many infarcts do not show on CT until hours after the onset of stroke. Ninety percent of large cortical infarcts are identifiable by CT $48 \mathrm{~h}$ after stroke onset compared with about $40 \%$ of lacunar or small cortical infarctions.

The majority of larger infarcts are visible within $6 \mathrm{~h}$. A typical sign of acute infarction on CT is hypo-attenuating brain tissue in an arterial territory and is thought to represent irreversible damage. During the initial $3 \mathrm{~h}$ of ischemia, the intracellular increase in water and sodium contents is almost exclusively confined to the gray matter. Brain cortex water content increases immediately after arterial occlusion. If brain water content increases by $1 \%$, CT attenuation decreases by 2-3 HU. Oedema in the ischemic cortex first diminishes its contrast with respect to the adjacent white matter and there is a loss of anatomic margins. Between 10 and 21 days, infarctions become isodense compared with normal brain; a finding that may last for several days and up to 2 weeks. This phase is called "fogging" and the extent of an infarction may be underestimated.[4] By 2-3 months, the infarctions are recognized as areas of CSF density and are easily visible.

One of the early CT findings in acute Middle Cerebral Artery (MCA) infarction is that of obscuration of lentiform nucleus, which is due to cellular oedema in the basal ganglia. A hyper attenuating MCA is another early sign of acute MCA infarction, representing acute thrombus within the M1 segment. Hypoattenuation on CT is highly specific for irreversible ischemic brain damage if it is detected within first 6 hours.[5]

Our study was done to ascertain the usefulness and yield of CT brain imaging in acute stroke, which will be helpful for us clinicians in thrombolysing patients at the earliest, so that the Morbidity and Mortality of acute cerebrovascular events is reduced.

Despite the limited sensitivity of non-contrast imaging in acute stroke, CT is still the best screening tool in view of wide availability as well as cost effectiveness in our country.

This study would give us an overall picture about the efficacy of non-contrast CT and its role in a tertiary setup for diagnosing acute stroke in patients presenting with neurological deficits.

\section{OBJECTIVES}

To assess the "YIELD AND USEFULNESS OF NON-CONTRAST CT-BRAIN IMAGING IN ACUTE STROKE" in a tertiary setup.

To establish the role of CT brain in acute stroke as an effective screening tool and correlation between the yield and time of presentation in acute stroke.

\section{METHODOLOGY}

Institutional Ethical Committee approval and informed consent from study subjects were obtained. This was a prospective observational study done from June 2015November 2015 in Government Stanley Hospital. This study is a prospective study, wherein 75 consecutive patients who presented with acute neurological deficit of vascular aetiology were studied. CT brain imaging was performed for all the patients. Analysis of images was done to come to a conclusion on using non-contrast CT imaging as a useful tool in patients with acute stroke and its effectiveness in picking up acute stroke.

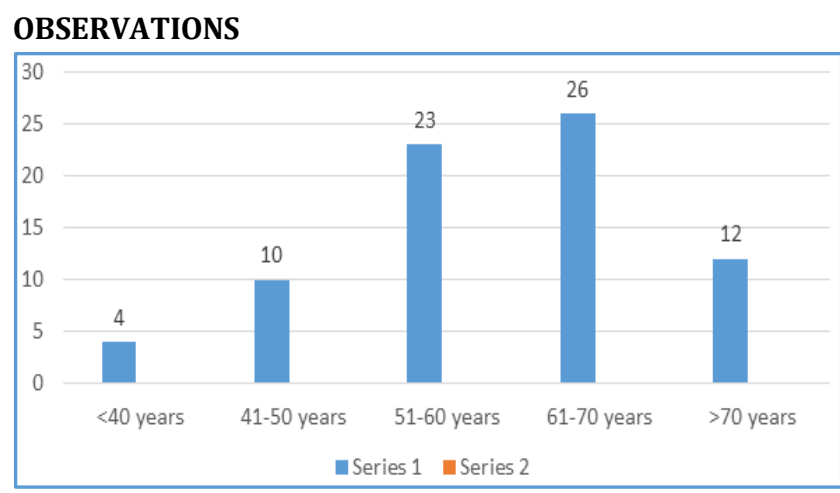

Fig. 1: Age Wise Distribution of Patients who Presented with Acute Stroke 


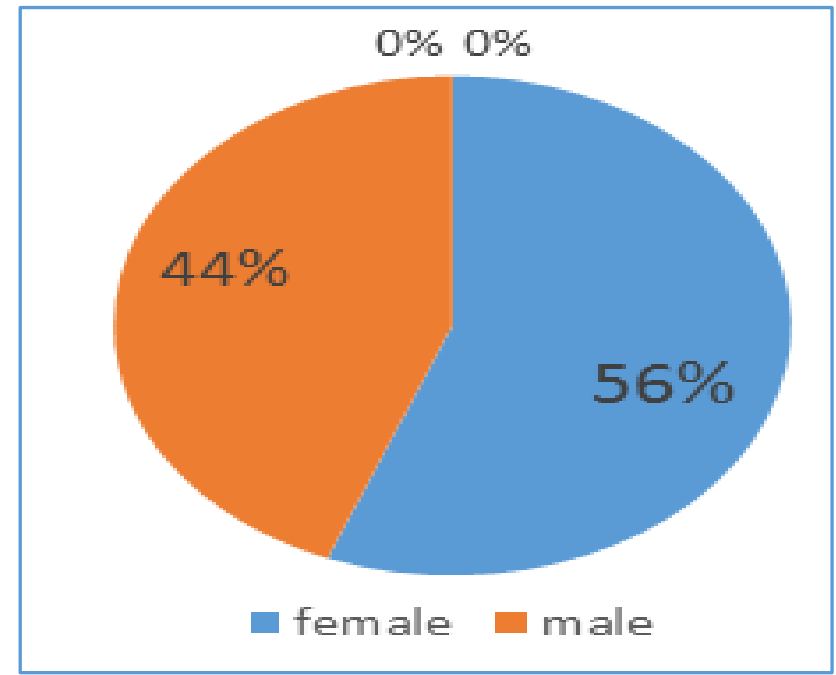

Fig. 2: PIE Chart Depicting Sex Wise Distribution

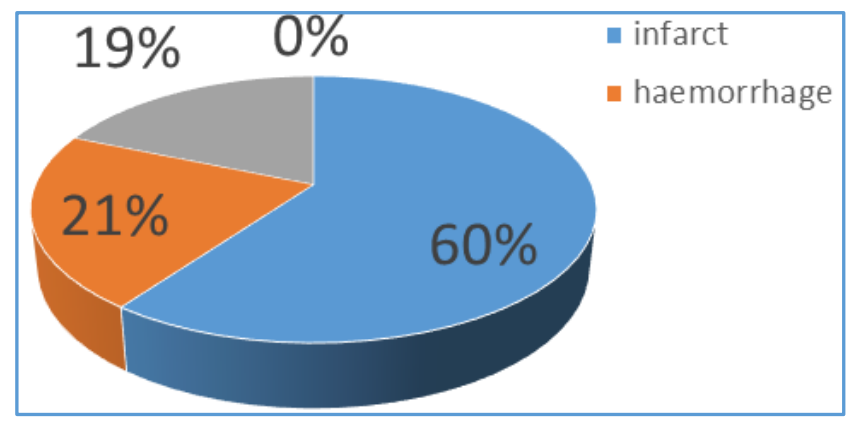

Fig. 3: PIE Chart Showing Pattern of CT Imaging in Acute Stroke

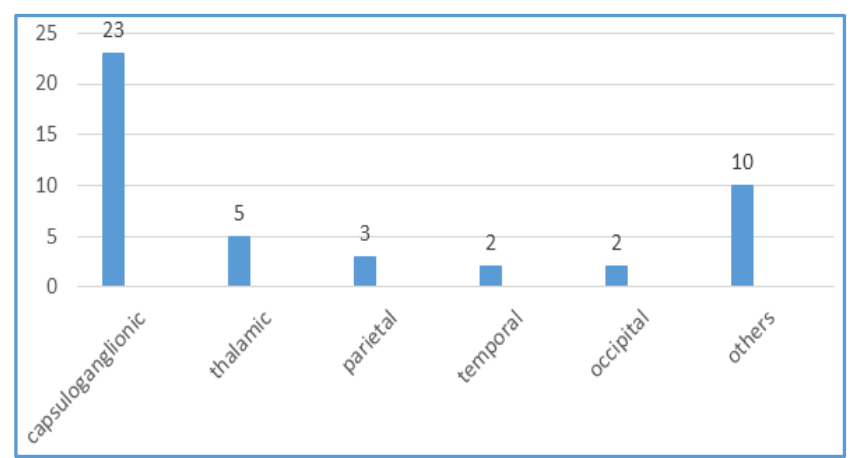

Fig. 4: Radiological Profile of Sites Involved in Acute Infarct

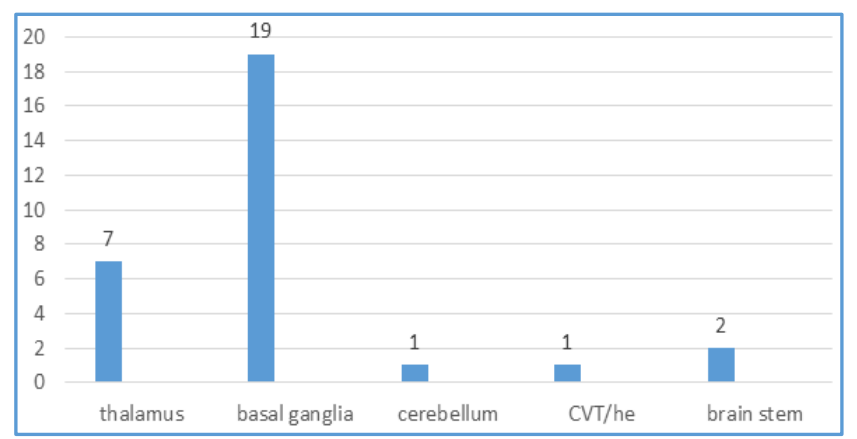

Fig. 5: Radiological Profile of Sites Involved-Haemorrhage

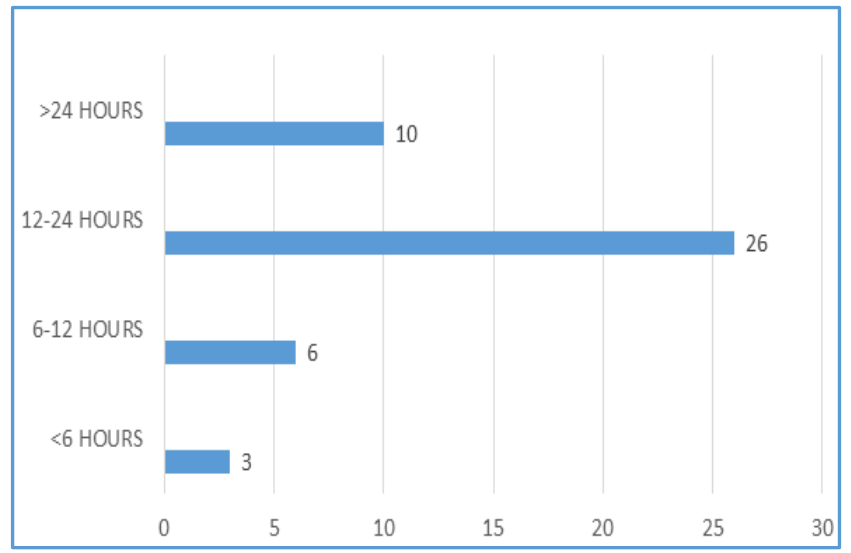

Fig. 6: Time of Presentation of Patients with CT Proven Infarct

\section{DISCUSSION}

CT is considered to be the gold standard for excluding ICH in the acute setting, although this role for CT has never been validated with neuropathological studies. Acute extravascular blood appears as hyperdense regions on the CT scan because of the high protein content of the haematoma.[6] CT can detect the effects of ischemia on brain tissue. Ischemia is a functional state of abnormal blood flow that initially leads to neuronal and endothelial cytotoxic oedema and subsequently to ionic oedema.[7] This increase in the water content of the brain causes X-ray attenuation and is seen as hypodensity on CT, most commonly in the arterial watershed territoriesnamely the insular cortex, the lentiform nucleus and the graywhite matter junction.

Early Ischemic Change (EIC) on NCCT is a term encompassing changes that almost certainly represent a number of different pathological processes in acute ischemia, whose significance varies. Previous radiological prejudice that CT within a few hours of stroke onset has low sensitivity is unfounded at least in Middle Cerebral Artery (MCA) occlusions, where EICs are present in around $70 \%$ of cases within three hours of onset. While the sensitivity of these changes is compromised by their subtlety, inter-observer reliability can be improved by systematic CT scan evaluation using systems such as the Alberta Stroke Programme Early CT Score (ASPECTS).[8]

\section{CT Findings in Acute Stroke}

The earliest CT sign visible is an hyperdense segment of a vessel representing direct visualisation of the intravascular thrombus/embolus and as such is visible immediately, although this can be seen in any vessel, it is most often observed in the middle cerebral artery (Hyperdense middle cerebral artery sign).

\section{Hypoattenuating Brain Tissue}

The reason we see ischemia on CT is that in ischemia cytotoxic oedema develops as a result of failure of the ionpumps. These fail due to an inadequate supply of ATP. An increase of brain water content by $1 \%$ will result in a CT attenuation decrease of $2.5 \mathrm{HU}$.

On the left, the patient with hypoattenuating brain tissue in the right hemisphere. The diagnosis is infarction because of the location (Vascular territory of the Middle Cerebral Artery (MCA) and because of the involvement of 
grey and white matter, which is also very typical for infarction.

\section{Obscuration of the Lentiform Nucleus}

Obscuration of the lentiform nucleus also called blurred basal ganglia is an important sign of infarction. It is seen in middle cerebral artery infarction and is one of the earliest and most frequently seen signs.[9]

\section{CONCLUSIONS}

Most common cause of stroke in our study was infarct (80\%); $19 \%$ amongst those stroke patients had no evidence of infarct at the time of presentation. Most common site of infarct was in the capsuloganglionic region (50\%). Only $10 \%$ of those who came before 6 hours had evidence of infarcts in CT when compared to $50 \%$ amongst those whose CT was taken after 12 hours. The lentiform nucleus was obscured in 8 patients with infarct, while the infarcted tissue showed hypoattenuation in 12 patients, dense MCA sign was seen in 10 patients. Patients who present with symptoms of stroke and who demonstrate hypodensity on CT within first six hours were proven to have larger infarct volumes, more severe symptoms.

Julio Chalela et al[10] stress that MRI is the current gold standard in imaging acute stroke. Indeed, MRI is an invaluable tool with which to visualize within minutes of cerebral infarction, what is believed to be the core (area that is already dead) and the penumbra (tissue at risk that might either die or survive).[11] By contrast, conventional CT has only limited use in detecting acute ischaemic stroke. However, the emergency assessment of most stroke patients still relies on CT alone Jeyadurai Pandian et al from CMC Ludhiana also reported the incidence of infarcts in acute stroke across 4 cities in India to be around $80 \%$, which correlates with our studies.[12] Radiological yield of evidence of infarct in a plain CT had positive correlation with advancing age of the infarct.

What is the "optimal time" to image the stroke patient?

There is no "optimal time" and the "best time" is as soon as possible. In routine practice, the timing is determined by the treatments that are considered as well as the imaging resources that are available. In patients who are considered candidates for intravenous or intra-arterial thrombolysis, CT is necessary to exclude haemorrhage or a sizeable infarction before the therapy is started. In acute setting, CT is helpful to differentiate vascular from non-vascular aetiologies for infarction and to exclude haemorrhage. Disadvantages of CT are that only $50 \%$ of infarcts will become visible that there is no "optimal time" for "seeing" an infarct and "seeing" the infarct is not necessary to diagnose ischemic stroke.

Nevertheless, with the addition of perfusion, CT becomes a very powerful technique in the diagnosis of acute infarctions. The optimal method for infarct detection should be rapid and cost-effective in the diagnosis and serve to triage the patients to the effective therapy. Such technique should be readily available, take no more than a few minutes to perform, be accurate and precise.

Thus, in cases of hyperacute stroke (0-6 h), CT is usually not sensitive in the identification of cerebral infarction. But it is quite sensitive in identifying various forms of acute intracranial haemorrhage and other gross lesions that would preclude the use of thrombolytic therapy. At a minimum, the presence of extensive CT abnormalities in a patient presenting in the first 6 hours for possible thrombolysis should necessitate a careful review of the time of stroke onset and the timeframe within which CT scan is taken also plays an important role in picking up acute infarct along with the site involved, which would give us a clue regarding the culprit vessel.

\section{REFERENCES}

1. Michael H Lev, Jeffrey Farkas, Joseph J Gemmete, et al. Acute stroke: improved nonenhanced ct detection benefits of soft-copy interpretation by using variable window width and center level settings. Radiology 1999;213(1):150-5.

2. Pressman BD, Tourje EJ, Thompson JR. An early CT sign of ischemic infarction: increased density in a cerebral artery. AJR Am J Roentgenol 1987;149(3):583-6.

3. Rowley HA. The four Ps of acute stroke imaging: parenchyma, pipes, perfusion, and penumbra. AJNR 2001;22:599-601.

4. Becker $\mathrm{H}$, Desch $\mathrm{H}$, Hacker $\mathrm{H}$, et al. CT fogging effect with ischemic cerebral infarcts. Neuroradiology 1979;18(4):185-92.

5. Rudiger von Kummer, Holger Bourquain, Stefano Bastianello, et al. Early prediction of irreversible brain damage after ischemic stroke at CT. Radiology 2001;219(1):95-100.

6. Kucinski T, Koch C, Zeumer H. In imaging in stroke. ed: Hennerici MG. Remedica publishing, London, 2003;1942.

7. Marc Simard J, Thomas A Kent, Mingkui Chen, et al. Brain oedema in focal ischaemia: molecular pathophysiology and theoretical implications. Lancet Neurology 2007;6(3):258-68.

8. Barber PA, Demchuk AM, Zhang J, et al. Validity and reliability of a quantitative computed tomography score in predicting outcome of hyperacute stroke before thrombolytic therapy. ASPECTS study group. Alberta stroke programme early CT score. Lancet 2000;355(9216):1670-4.

9. Tomura N, Uemura K, Inugami A. Early CT finding in acute stroke: obscuration of lentiform nucleus. Radiology 1988;168(2):463-7.

10. Chalela JA, Kidwell CS, Nentwich LM, et al. Magnetic resonance imaging and computed tomography in emergency assessment of patients with suspected acute stroke: a prospective comparison. Lancet 2007;369(9558):293-8.

11. Hjort N, Christensen S, Solling C, et al. Ischemic injury detected by diffusion imaging 11 minutes after stroke. Ann Neurol 2005;58(3):462-5.

12. Jeyaraj Pandian, Paulin Sudhan. Stroke epidemiology and stroke care services in India. Journal of stroke 2013;15(3):128-34. 